\title{
The F. and M. Riesz theorem and group structures
}

\author{
By Shozo Koshi and Hiroshi Yamaguchi* \\ (Received April 10, 1979)
}

\section{Introduction}

In [4] and [5], one of the authors of the present note considered the relation between the F. and M. Riesz theorem and the structures of locally compact abelian (LCA) groups. In this note, we shall refine these results in a more general setting.

Let $G$ be a LCA group with the algebraically ordered dual $\hat{G}=\Gamma$. If there exists a semi-group $P$ of $\Gamma$ such that

$$
\begin{aligned}
& P \cup(-P)=\Gamma \\
& P \cap(-P)=\{0\},
\end{aligned}
$$

then $\Gamma$ is called algebraically ordered, and $P$ is called a semi-group with $(\mathrm{AO})$-conditions.

Let $M_{P}^{a}(G)$ be the set of all analytic measures with respect to $P$. Here a measure $\mu$ on $G$ is analytic $w . r$. to $P$ if and only if the Fourier transformation $\hat{\mu}$ is vanishing outside $P . \quad L^{1}(G)$ denotes the space of all integrable functions with respect to the Haar measure on $G$.

The main result is: If $0 \neq M_{P}^{a}(G) \subset L^{1}(G)$, then $G$ must be isomorphic to one of the following

$$
R \times D \quad \text { or } T \times D
$$

where $R$ is the reals, $T$ the torus and $D$ some devisible discrete abelian group.

We express our hearty thanks to Professor J. Inuoue who has given important ideas in order to prove the theorems.

\section{Main theorems}

Let $G$ be a LCA group with the algebraically ordered dual $\Gamma=\hat{G}$ so that there exists a semi-group $P$ of $\Gamma$ with (AO)-conditions. Then $\Lambda=\bar{P} \cap(\overline{-P})$ is a closed subgroup of $\Gamma$. It is easy to see that $\Lambda$ is the boundary of $P$ and $-P$ respectively.

\footnotetext{
* H. Yamaguchi is the same person as H. Otaki.
} 
Lemma 1. Let $F$ be a compact subgroup of $\Gamma$. Then $A$ contains $F$.

Proof. $F \cap P$ is a semigroup with (AO)-conditions in $F$. Since $F$ is compact, $F \cap P$ is dense in $F$. (cf. Lemma 1 of [5]). Hence, $F \subset \bar{P}$ and similarly $F \subset(\overline{-P})$.

Lemma 2. Let $\gamma \in \Gamma \backslash \Lambda$. Then, there exists an open set $V \ni \gamma$ with $V+\Lambda \subset P \backslash\{0\}$ or $V+\Lambda \subset-P \backslash\{0\}$.

Proof. It $\gamma \in P \backslash A$, then there exists an open set $V \ni \gamma$ such that $V \subset$ $P \backslash \Lambda$. In this case, we have $V+\Lambda \subset P \backslash\{0\}$. If $\gamma \in-P \backslash \Lambda$, then there exists an open set $V \ni \gamma$ with $V+\Lambda \subset-P \backslash\{0\}$. q. e. d.

Lemma 3. Let $\tilde{P}=\{[\gamma] ;[\gamma]=\gamma+\Lambda \subset P\} \cup[0] . \quad$ Then, $\tilde{P}$ is a closed semigroup with $(A O)$-conditions in $\Gamma / \Lambda$. Moreover $\pi(P)=\tilde{P}$ and $\pi(-P)=-\tilde{P}$ where $\pi$ is a natural homomorphism $\Gamma \rightarrow \Gamma / \Lambda$.

This is an easy consequence of Lemma 2.

Lemma 4. If $\{0\} \neq M_{P}^{a}(G) \subset L^{1}(G)$, then $\Gamma / \Lambda$ is isomorphic to $R$ or $Z$.

Proof. If $\Gamma / \Lambda$ is not isomorphic to $R$ or $Z$, then there exists a measure $\mu$ on $(\widehat{\Gamma / \Lambda})=\Lambda^{\perp}$ (annihilator of $\Lambda$ ) with $\mu \in M_{\tilde{\vec{P}}}^{a}\left(\Lambda^{\perp}\right)$ and $\hat{\mu} \notin C_{\mathrm{o}}(\Gamma / \Lambda)$ (cf. [4]). Since $\mu$ is considered as a measure on $G$, and the Fourier transform $\hat{\mu}$ is vanishing outside $P$ and $\tilde{\mu} \notin C_{0}(\Gamma)$ by Lemma 3 , we have a contradicition.

Lemma 5. Under the assumption of Lemma $4, \Gamma \cong \Lambda \times R$ or $\Gamma \cong \Lambda \times Z$.

Proof. By structure theorem ([3], (24.30) Theorem), $\Gamma \cong R^{k} \times F(k$ : integer) where $F$ contains an open compact subgroup. If $\Gamma / \Lambda \cong R$, then $R \cong \Gamma / \Lambda \supset\left(R^{k}+\Lambda\right) / \Lambda \cong R^{k} /\left(\Lambda \cap R^{k}\right)$. Since $S=R^{k} / \Lambda \cap R^{k}$ is isomorphic to a closed subgroup of $R$ and is not discrete, we have (i) $S \cong R$ or (ii) $S \cong 0$.

Assume (i) is occured, then $R^{k} \cong R \times\left(\Lambda \cap R^{k}\right)$. Hence, $\Gamma \cong R \times\left(\Lambda \cap R^{k}\right) \times F$. Since $\Lambda \cap[R \times\{0\} \times\{0\}]=\{0\}$, and $\Gamma / \Lambda \cong R$, we have $\Gamma \cong R \times \Lambda$.

In the case (ii), $\Lambda \supset R^{k}$ and so $\left.R \cong \Gamma / \Lambda \cong\left(\Gamma / R^{k}\right) /\left(\Lambda / R^{k}\right) \cong F / \Lambda / R^{k}\right)$. But, this is a contradiction, since $F$ has an open compact subgroup.

If $\Gamma / \Lambda \cong Z$, then it is clear that $\Gamma \cong \Lambda \times Z$.

We state now the main theorem.

THEOREM 1. Let $G$ a LCA group with the algebraically ordered dual $\Gamma=\hat{G}$, and let $P$ be a semi-group with $(A O)$-conditions of $\Gamma$. If $\{0\} \neq$ $M_{P}^{a}(G) \subset L^{1}(G)$, then $G$ is isomorphic to one of both $R \times D$ or $T \times D$ where $D$ is a divisible discrete group.

Proof. By Lemma $5, \Gamma \cong \Lambda \times H$ where $H \cong R$ or $Z$. We have only to prove that $\Lambda$ is compact. Remark now that $G=\hat{\Gamma} \cong H^{\perp} \times \Lambda^{\perp}, \hat{H}^{\perp}=\Lambda$ and $\left(\hat{\Lambda}^{\perp}\right)=H$. Assume that $\Lambda$ is not compact, then there exists a $\nu \in M\left(H^{\perp}\right)$ (measure on $H^{\perp}$ ) with $\hat{\nu} \notin C_{0}(\Lambda)$. By Lemma 2, there exists a $\gamma \in H$ and an 
open set $V \ni \gamma$ with $V+\Lambda \subset P \backslash\{0\}$. Hence, there exists a non zero $\rho \in L^{1}\left(\Lambda^{\perp}\right)$ with supp $\hat{\rho} \subset V \cap H$. Putting $\mu=\nu \times \rho$, we have

$$
\operatorname{supp} \hat{\mu} \subset A+V \subset P \text { and } \hat{\mu} \notin C_{0}(\Gamma) \text {. }
$$

Hence $\mu \in M_{P}^{a}(G) \backslash L^{1}(G)$. This is a contradiction.

REMARK 1. Theorem 1 in $([5])$ is an immediate consequence of Theorem 1 of this paper.

REMARK 2. Let $F$ be a compact abelian torsion-free group.

(1) If $P$ is a semigroup with (AO)-conditions of $R \times E$, and if $P$ is not dense in $R \times F$, then $P$ is either

$$
\left\{(x, f) \in R \times F ; x>0, \text { or } x=0 \text { and } f \geqq{ }_{P} 0\right\}
$$

or

$$
\left\{(x, f) \in R \times F ; x<0, \text { or } x=0 \text { and } f \geqq_{P} 0\right\} \text {. }
$$

Here ' $>$ ' denotes the usual order of $R$ and ' $\geqq_{P}$ ' denotes the order of $F$ induced by the semigroup $P$.

(2) Let $P$ be a semigroup with (AO)-conditions of $Z \times F$. If $P$ is not dense in $Z \times F$, then $P$ is either

$$
\left\{(n, f) \in Z \times F ; n>0, \text { or } n=0 \text { and } f \geqq{ }_{P} 0\right\}
$$

or

$$
\left\{(n, f) \in Z \times F ; n<0, \text { or } n=0 \text { and } f \geqq_{P} 0\right\} \text {. }
$$

Theorem 2. Suppose $G$ is one of both $T \times D$ and $R \times D$, where $D$ is a discrete abelian group such that $\hat{D}$ is torsion-free. Assume that $P$ is a semigroup of $\hat{G}$ with $(A O)$-conditions such that it is not dense in $\hat{G}$. Then, $M_{P}^{a}(G) \subset L^{1}(G)$.

Indeed, when $G$ is either $T$ or $R$ this is the $F$. and $M$. Riesz theorem. The proof is given in Proposition $A$ of [5] for $G=T \times D$. The latter case can be proved in the same way as the case $G=T \times D$.

\section{Denseness of a semigroup with (AO)-conditions}

An abelian group $G$ is algebraically ordered if and only if it is torsionfree, since every group $G$ can be considered as a discrete group (c.f. 8.1. 2 of [6]). Therefore, these two terms are synonymous.

Proposition 1. Let $R$ be the additive group of the real numbers. 
There exists a dense subset of which is a semigroup with $(A O)$-conditions.

PRoOF. Let $\left\{e_{\lambda}\right\}_{x \in A}$ be a Hamel basis of $R$ with respect to the rational number field. We can assume that every $e_{2}$ is positive. We introduce a linear order in $\Lambda$. Every $x \in R$ is written into the following:

$$
x=\sum_{k=1}^{n} \alpha_{k} e_{\lambda_{k}} ; \lambda_{1}>\lambda_{2}>\cdots>\lambda_{n},
$$

where $\alpha_{k}$ is a rational number for $k=1,2, \cdots, n$. Let $P=\left\{x ; x=\sum_{k=1}^{n} \alpha_{k} e_{\lambda_{k}}\right.$; $\lambda_{1}>\lambda_{2}>\cdots>\lambda_{n}$ and $\left.\alpha_{1}>0\right\} \cup\{0\}$.

Then it is easy to see that $P$ is a semigroup with (AO)-conditions in $R$. An order induced by $P$ is known as the lexicographic order. But $P$ contains not only positive numbers, but also negative numbers. So, it is easy see that $P$ is dense in $R$ by the usual topology.

Proposition 2. Let $G_{1}$ and $G_{2}$ be LCA groups with semigroups $P_{1}$ and $P_{2}$ with $(A O)$-conditions. If $P_{1}$ is dense in $G_{1}$, then there exists in $G=G_{1} \times G_{2}$ a dense semigroup $P$ reith $(A O)$-conditions. If $P_{1}$ is not dense in $G_{1}$, then there exists a semigroup $P$ with $(A O)$-conditions which is not dense in $G=G_{1} \times G_{2}$.

PRoof. We construct a linear order by lexicographic method in $G=$ $G_{1} \times G_{2}$ so that $g_{1}+g_{2} \geqq g_{1}^{\prime}+g_{2}^{\prime}$ means that $g_{1}-g_{1}^{\prime} \in P_{1} /\{0\}$ or $\left\{g_{1}=g_{1}^{\prime}, g_{2} \geqq g_{2}^{\prime}\right\}$. Then the semigroup induced by this linear order satisfies above conditions.

THEOREM 3. Let $\Gamma$ be a nondiscrete locally compact abelian torsionfree group. Then there exists a dense subset which is a semigroup with (AO)-conditions.

Proof. By structure theorem ([3], (24.30) Theorem), $\Gamma \cong R^{n} \times F$, where $n$ is a nonnegative integer and $F$ is a LCA group with a compact open subgroup $F_{0}$. By Propositions 1 and 2 , we can easily construct a semigroup with the required condition if $n \geqq 1$. Therefore we will prove the case $n=$ 0 , i.e, $\Gamma \cong F$. The proof of this case calls for Zorn's lemma. Since $\Gamma$ is not discrete, it follows that $F_{0} \neq\{0\}$.

Let $\mathscr{F}$ denote the set of the following pairs $(\Lambda, P): \Lambda$ is an open subgroup which includes $F_{0}$ as a subgroup. $P$ is a dense subset of $\Lambda$ which is a semigroup with (AO)-conditions. $\mathscr{F}$ is not empty because $F_{0}$ has such a semigroup. For $\left(\Lambda_{1}, P_{1}\right),\left(\Lambda_{2}, P_{2}\right) \in \mathscr{F}$, define $\left(\Lambda_{1}, P_{1}\right) \leqq\left(\Lambda_{2}, P_{2}\right)$ if and only if $\Lambda_{1} \subset \Lambda_{2}$ and $P_{1} \subset P_{2}$. By this " $\leqq$ ", $\mathscr{F}$ is partially ordered.

Let $\left.\left\{\Lambda_{\alpha}, P_{\alpha}\right)\right\}_{\alpha \in I}$ be a totally ordered subset of $\mathscr{F}$. Let $\Lambda_{0}$ and $P_{0}$ denote $\bigcup_{\alpha \in I} \Lambda_{\alpha}$ and $\bigcup_{\alpha \in I} P_{\alpha}$ respectively. Then $\Lambda_{0}$ is an open subgroup of $\Gamma$ and $F_{0} \subset$ 
$\Lambda_{0}$. It is easy to check that $P_{0}$ is a semigroup with (AO)-conditions and $P_{0}$ is dense in $\Lambda_{0}$. Thus, $\left(\Lambda_{0}, P_{0}\right) \in \mathscr{F}$ and $\left(\Lambda_{\alpha}, P_{\alpha}\right)<\left(\Lambda_{0}, P_{0}\right)$ for every $\alpha \in I$. By Zorn's lemma, there exists a maximal element $\left(\Lambda_{*}, P_{*}\right) \in \mathscr{F}$. It is sufficient to show $\Lambda_{*}=\Gamma$ in order to prove the theorem. We suppose $\Lambda_{*} \sqsubseteq \Gamma$ and derive a contradiction. First, consider the case that $\Gamma / \Lambda_{*}$ is not a torsion group. There exists an element $\gamma_{0} \in \Gamma$ (but $\gamma_{0} \notin \Lambda_{*}$ ) such that $n \gamma_{0}+$ $\Lambda_{*} \neq \Lambda_{*}$ for every natural number $n$. Thus, the open subgroup $\left[\gamma_{0}, \Lambda_{*}\right]$ generated by $\gamma_{0}$ and $\Lambda_{*}$ is isomorphic to $Z \times \Lambda_{*}$. Let $P=\left\{(n, \gamma) \in\left[\gamma_{0}, \Lambda_{*}\right]\right.$; $\left.\gamma \in P_{*} \backslash\{0\}, n \in Z\right\} \cup\left\{(n, 0) \in\left[\gamma_{0}, \Lambda_{*}\right] ; n \geqq 0\right\}$. Then $P$ is a semigroup with (AO)-conditions which is dense in $\left[\gamma_{0}, \Lambda_{*}\right]$. That is, $\left(\left[\gamma_{0}, \Lambda_{*}\right], P\right)$ belongs to $\mathscr{F}$ and $\left(\Lambda_{*}, P_{*}\right) \leqq\left(\left[\gamma_{0}, \Lambda_{*}\right], P\right)$. This contradicts the maximality of $\left(\Lambda_{*}, P_{*}\right)$. If $\Gamma / \Lambda_{*}$ is a torison group, define a semigroup $P$ as follows :

$P=\left\{\gamma \in \Gamma ; n \gamma \in P_{*}\right.$ for some positive integer $n$ satisfying $\left.n \gamma+\Lambda_{*}=\Lambda_{*}\right\}$. Then, since $\Gamma$ is torsion-free, $P$ is a semigroup with (AO)-conditions. Since $P \supset P_{*}$ and $P_{*}$ is dense in $\Lambda_{*}, \bar{P} \cap \overline{(-P)} \supset \Lambda_{*}$. If we suppose that $P$ is not dense in $\Gamma$, there exists $\gamma \in \Gamma$ with $\gamma+\Lambda_{*} \subset P$ by Lemma 2. Since there exists a positive number $n$ with $n \gamma \in \Lambda_{*}$, it follows that $P_{*}=\Lambda_{*} \cap P$ $\supset n\left(\gamma+\Lambda_{*}\right)=\Lambda_{*}$. This is impossible. Hence, $P$ is dense in $\Gamma$. This implies that $(\Gamma, P) \in \mathscr{F}_{*}$ and $\left(\Lambda_{*}, P_{*}\right) \leqq(\Gamma, P)$, contradicting the maximality of $\left(\Lambda_{*}, P_{*}\right)$.

Q. E. D.

Proposition 3. Suppose $\Gamma$ is a locally compact abelian torsion-free group and $\Gamma \cong R^{n} \times F$, where $n$ is a nonnegative integer and $F$ is a LCA group which has a compact open subgroup $F_{0}$. Then there is a semigroup $P$ with $(A O)$-conditions of $\Gamma$ which is not dense in $\Gamma$ in $n \geqq 1$.

In case $n=0$, such a semigroup exists if and only if $\Gamma / F_{0}$ is not a torsion group.

Proof. If $n \geqq 1$, it is easy to construct a such semigroup $P$ by proposition 1. Thus we consider the case $n=0$. If $\Gamma / F_{0}$ is a torsion group, then a semigroup $S$ with (AO)-conditions of $\Gamma$ is always dense in $\Gamma$ by the fact that every semigroup with (AO)-conditions in a compact group is always dense, because for every $\gamma \in \Gamma$ there exists an integer $n$ such that $\left\{\gamma+F_{0}\right\} \cup$ $\left\{z \gamma+F_{0}\right\} \cup \cdots \cup\left\{n \gamma+F_{0}\right\}$ is a compact open subgroup of $\Gamma$. Hence, $\Gamma / F_{0}$ is not a torsion group if there exists a semigroup $P$ with (AO)-conditions not dense in $\Gamma$. Now, we prove the converse.

Let $\mathscr{F}=\left\{\gamma+F_{0} ; O\left(\gamma+F_{0}\right)<\infty\right.$ in $\left.\Gamma / F_{0}\right\}$ where $O\left(\gamma+F_{0}\right)$ denotes the order of the coset in $\Gamma / F_{0}$, and let $F_{0 ; \%}=\cup\left\{\gamma+F_{0} ; \gamma+F_{0} \in \mathscr{F}\right\}$. By the hypothesis, $F_{0, \approx} \neq \Gamma$. Furthermore, $\Gamma / F_{0, \xi}$ is torsion-free, as we can prove in the following way. Suppose that there exist $\gamma_{0} \notin F_{0, \mathscr{F}}$ and an integer $n>1$ such that $n \gamma_{0}+F_{0, \mathscr{F}}=F_{0, \mathscr{\xi} \text {. }}$ Then $n \gamma_{0}+F_{0}=\gamma+F_{0}$ for some $\gamma+F_{0} \in \mathscr{F}$, 
and $m n \gamma_{0}+F_{0}=F_{0}$ where $m=O\left(\gamma+F_{0}\right)$ in $\Gamma / F_{0}$. This implies $\gamma_{0}+F_{0} \in \mathscr{F}$. This is a contradiction.

Now, we can take a semigroup $P_{1}$ with $(\mathrm{AO})$-conditions of $F_{0, \mathscr{s}}$, since $F_{0, \mathscr{F}}$ is a torsion free group.

Since $\Gamma / F_{0, \mathscr{\xi}}$ is torsion-free, there exists a semigroup $P_{2}$ with (AO)conditions of $\Gamma / F_{0, \mathfrak{w}}$.

Let $P=\left\{\gamma \in \Gamma ;[\gamma] \in P_{2} \backslash\{[0]\}\right\} \cup P_{1}$, where $[\gamma]$ denotes the coset of $\gamma$ in $\Gamma / F_{0, \mathscr{F}}$. Then $P$ is a semigroup with $(\mathrm{AO})$-conditions which is not dense in $\Gamma$.

Q. E. D.

\section{References}

[1] Doss, R.: On measures with small transforms. Pacific Journal of Math. 26, 257-263 (1968).

[2] Helson, H. and Lowdenslager, D.: Prediction theory and Fourier series in several variables. Acta Math. 99, 165-202 (1958).

[3] Hewitt, E. and Ross, K. A.: Abstract Harmonic Analysis Vol 1. BerlinHeidelberg-New York: Springer 1963.

[4] OTAKI, H.: Remarks on characterization of locally compact abelian groups. Hokkaido Math. J. 6, 52-55 (1977).

[5] OTAKI, H.: A ralation between the F. and M. Riesz theorem and the structure of LCA groups. Hokkaido Math. J. 6, 306-312 (1977).

[6] Rudin, W.: Fourier Analysis on Groups. New York-London: Interscience 1962. 